\title{
A FURTHER REFINEMENT OF THE BRUHAT DECOMPOSITION
}

\author{
CHARLES W. CURTIS
}

(Communicated by Bhama Srinivasan)

\begin{abstract}
Kawanaka obtained explicit formulas for the structure constants in the Hecke algebra $H(G(q), B(q))$ of a finite Chevalley group $G(q)$. This note contains a geometric interpretation of these formulas, involving decompositions of Bruhat cells, in connected reductive algebraic groups.
\end{abstract}

Introduction. Let $G$ be a connected reductive affine algebraic group over an algebraically closed field $k$, defined and split over a perfect subfield $k_{0} \subseteq k$. Let $W=$ $N_{G}(T) / T$ be the Weyl group of $G$ with respect to a fixed maximal split torus $T$, contained in a Borel subgroup $B$, defined and split over $k_{0}$. Let $\left(w, w^{\prime}, w^{\prime \prime}\right)$ denote an arbitrary triple of elements of $W$, and let $\dot{w}, \dot{w}^{\prime}, \dot{w}^{\prime \prime}$ denote their representatives in $N_{G}(T)\left(k_{0}\right)$. Let $U=R_{u}(B)$, and let $U_{x}=U \cap{ }^{x} B_{-}$for $x \in W$, where $B_{-}$ is the Borel subgroup opposed to $B$. The main result in this note is an explicit decomposition of the set

$$
U\left(w, w^{\prime}, w^{\prime \prime}\right)=\left\{u \in U_{w}: u \dot{w} B \cap \dot{w}^{\prime \prime} U_{\left(w^{\prime}\right)^{-1}}\left(\dot{w}^{\prime}\right)^{-1} \neq \varnothing\right\}
$$

as a disjoint union of subsets $\left\{U_{\tau}\right\}$ indexed by certain subexpressions $\tau$ of a reduced expression of $w$ as a product of the distinguished generators $\left\{s_{1}, \ldots, s_{n}\right\}$ of $W$. Each subset $U_{\tau}$ is a locally closed $k_{0}$-subvariety of $U_{w}$ and is isomorphic, as a variety defined over $k_{0}$, to a product $G_{a}^{a(\tau)} \times G_{m}^{b(\tau)}$, where $G_{a}$ and $G_{m}$ denote the additive and multiplicative groups of $k$, respectively, and $a(\tau)$ and $b(\tau)$ are nonnegative integers defined by the subexpression $\tau$.

In case $w^{\prime}=w_{0}$, the element of $W$ of maximal length, a similar problem was solved by Deodhar [3]. In the general case, the result gives a geometric interpretation of a result of Kawanaka [4]. He considered the situation where $k_{0}$ is a finite field, and proved that the structure constant $\left[b_{w} b_{w^{\prime}}: b_{w^{\prime \prime}}\right]$ for standard basis elements of the Iwahori algebra (or Hecke algebra) $H\left(G\left(k_{0}\right), B\left(k_{0}\right)\right)$ is given by

$$
\left[b_{w} b_{w^{\prime}}: b_{w^{\prime \prime}}\right]=\sum_{\tau} q^{a(\tau)}(q-1)^{b(\tau)} .
$$

A corollary of our result gives a description of each set $U_{\tau}\left(k_{0}\right)$ in terms of root subgroups of $G\left(k_{0}\right)$, which implies Kawanaka's formula, and will be applied in a subsequent paper to describe the multiplication in the Hecke algebra of the GelfandGraev representation of $G\left(k_{0}\right)$, in case $k_{0}$ is a finite field, and the center of $G$ is

Received by the editors September 2, 1986.

1980 Mathematics Subject Classification (1985 Revision). Primary 20G15.

Key words and phrases. Reductive algebraic groups, Bruhat decomposition, Hecke algebra.

The author's research was supported in part by NSF grant DMS-8318037-01. 
connected. Another interesting problem would be to describe the closure patterns of the subsets $\left\{U_{\tau}\right\}$.

The author thanks N. Kawanaka and V. Deodhar for discussions, at various times, on these ideas. The idea of seeking such a decomposition of the sets $U\left(w, w^{\prime}, w^{\prime \prime}\right)$ comes from the author's attempt to understand the structure of the Hecke algebra of the Gelfand-Graev representation of $G\left(k_{0}\right)$ for a finite field $k_{0}$ (see Yokonuma [6], Steinberg [5], and Chang [2]).

1. Statements of results. Besides the notation given in the Introduction, we require some other basic facts (for proofs, see Borel and Tits [1]). Let $\Phi$ denote the root system of $G$ with respect to $T,\left\{U_{\alpha}: \alpha \in \Phi\right\}$ the root subgroups, and $\Phi^{ \pm}$the sets of positive and negative roots defined by the Borel subgroups $B$ and $B^{-} . \Delta=\left\{\alpha_{i}: 1 \leq i \leq n\right\}$ denotes the set of simple roots in $\Phi^{+}$, corresponding to the set of fundamental reflections $S=\left\{s_{i}: 1 \leq i \leq n\right\}$. For each $x \in W$, let $\psi_{x}=\Phi^{+} \cap x \Phi^{-}$; then $\left|\psi_{x}\right|=l(x)$, where $l(x)$ is the length of $x$ in the Coxeter system $(W, S)$. We have

$$
U_{x}=\prod_{\alpha \in \psi_{x}} U_{\alpha}
$$

for an arbitrary ordering of the roots in $\psi_{x}$, and, in any order, the map of the direct product of the $\left\{U_{\alpha}, \alpha \in \psi_{x}\right\}$ to $U_{x}$ is an isomorphism of $k_{0}$-varieties. Moreover,

$$
U=U_{x} U_{x w_{0}}=U_{x w_{0}} U_{x}
$$

and the map from the direct product $U_{x} \times U_{x w_{0}}$ to $U$ in an isomorphism of $k_{0^{-}}$ varieties.

We have

$$
G=\bigcup_{x \in W} U_{x} \dot{x} B
$$

for a fixed cross section $\{\dot{x}: x \in W\}$ in $N_{G}(T)\left(k_{0}\right)$, and for each $x$ there exists an isomorphism of $k_{0}$-varieties $U_{x} \times B \cong U_{x} \dot{x} B$ given by the product map. A decomposition similar to (1.3) holds for the group of rational points $G\left(k_{0}\right)$.

Now let $\left(w, w^{\prime}, w^{\prime \prime}\right)$ denote an arbitrary triple of elements of $W$, which will remain fixed, and let $s_{l} \cdots s_{1}$ be a fixed reduced expression of $w$ with factors $s_{i} \in S$. We define

$$
U\left(w, w^{\prime}, w^{\prime \prime}\right)=\left\{u \in U_{w}: u \dot{w} B \cap \dot{w}^{\prime \prime} U_{\left(w^{\prime}\right)^{-1}}\left(\dot{w}^{\prime}\right)^{-1} \neq \varnothing\right\} .
$$

As in Deodhar [3], a subexpression $\tau$ of the reduced expression $s_{l} \cdots s_{1}$ is a sequence $\tau=\left(\tau_{l}, \ldots, \tau_{1}, \tau_{0}\right)$ of elements of $W$ with the property that $\tau_{i} \tau_{i-1}^{-1} \in\left\{1, s_{i}\right\}$ for $1 \leq i \leq l$ and $\tau_{0}=1$. Thus the set of terminal elements $\left\{\tau_{l}\right\}$ of subexpressions of $s_{l} \cdots s_{1}$ coincides with the set of elements $x \in W$ such that $x \leq w$ in the Bruhat order (see Deodhar [3]). A subexpression $\tau=\left(\tau_{l}, \ldots, \tau_{1}, \tau_{0}\right)$ is called a $K$-sequence relative to the triple $\left(s_{l} \cdots s_{1}=w, w^{\prime} w^{\prime \prime}\right)$ if it satisfies the conditions $(2.10)(\mathrm{a} \cdot \mathrm{c})$ of Kawanaka [4]. Let $J_{\tau}=\left\{j: \tau_{j} \tau_{j-1}^{-1}=s_{j}\right\} \cup\{0\}$. Then $\tau$ is such a $K$-sequence if and only if

$$
\tau_{l} w^{\prime}=w^{\prime \prime}, \quad l\left(s_{p} \tau_{j} w^{\prime}\right)<l\left(\tau_{j} w^{\prime}\right)
$$


for each $j \in J_{\tau}$ and $p$ in the interval between $j$ and the next element in $J_{\tau}$ (or simply all $p>j$ if $j$ is the maximal element of $J_{\tau}$ ). For each $K$-sequence $\tau$, set

$$
J_{\tau}^{-}=\left\{j \in J_{\tau}: l\left(s_{j} \tau_{j}, w^{\prime}\right)<l\left(\tau_{j}, w^{\prime}\right)\right\},
$$

where $j^{\prime} \in J_{\tau}$ is the predecessor of $j$, and define

$$
a(\tau)=\left|J_{\tau}^{-}\right|, \quad b(\tau)=l-\left|J_{\tau}\right|+1=\operatorname{card}\left\{j>0: \tau_{j} \tau_{j-1}^{-1}=1\right\} .
$$

The main results can be stated as follows.

(1.6) THEOREM. Let $\left(w, w^{\prime}, w^{\prime \prime}\right)$ be a triple of elements of $W, s_{l} \cdots s_{1}$ a fixed reduced expression of $w$, and $K=K\left(s_{l} \cdots s_{1}, w^{\prime}, w^{\prime \prime}\right)$ the set of $K$-sequences relative to $\left(s_{l} \cdots s_{1}, w^{\prime}, w^{\prime \prime}\right)$. Then $U\left(w, w^{\prime}, w^{\prime \prime}\right)$ is independent of the choice of the cross section $\{\dot{x}\}$, and we have

$$
U\left(w, w^{\prime}, w^{\prime \prime}\right)=\bigcup_{\tau \in K} U_{\tau} \quad \text { (disjoint union), }
$$

where, for each $\tau, U_{\tau}$ is a locally closed $k_{0}$-subvariety of $U_{w}$. Moreover, there exist subsets $\Phi_{\tau}$ and $\Phi_{\tau}^{*}$ of $\Phi^{+}$of cardinalities a $(\tau)$ and $b(\tau)$ respectively, such that, for each $\tau$, there exists an isomorphism of $k_{0}$-varieties

$$
U_{\tau} \cong \prod_{\alpha \in \Phi_{\tau}} U_{\alpha} \times \prod_{\beta \in \Phi_{\tau}^{*}} U_{\beta}^{*}
$$

(where $U_{\beta}^{*}$ denotes the open set $U_{\beta}-\{1\}$ for $\beta \in \Phi$ ).

In the statement of the next result, $U\left(w, w^{\prime}, w^{\prime \prime}\right)\left(k_{0}\right)$ and $U_{\tau}\left(k_{0}\right)$ denote the sets of $k_{0}$-rational points on $U\left(w, w^{\prime}, w^{\prime \prime}\right)$ and $U_{\tau}$, respectively, for $\tau \in K$.

(1.7) COROLlaRY. Keep the notation of Theorem 1.6. Then

$$
U\left(w, w^{\prime}, w^{\prime \prime}\right)\left(k_{0}\right)=\bigcup_{\tau \in K} U_{\tau}\left(k_{0}\right) \quad \text { (disjoint union), }
$$

where for each $\tau$, there exists a bijection

$$
U_{\tau}\left(k_{0}\right) \rightleftharpoons \prod_{\alpha \in \Phi_{\tau}} U_{\alpha}\left(k_{0}\right) \times \prod_{\beta \in \Phi_{\tau}^{*}} U_{\beta}^{*}\left(k_{0}\right) .
$$

2. Proofs. For each simple root $\alpha_{i} \in \Delta$, let $G_{i}$ denote the $k_{0}$-subgroup of semisimple rank one generated by $\left\{T, U_{ \pm \alpha_{i}}\right\}$. We require the following structure equations concerning the multiplication in $G_{i}$ :

(2.1) LEMMA. For each $i, 1 \leq i \leq n$, there exist automorphisms of $k_{0}$-varieties $f_{i}, g_{i}: U_{\alpha_{i}}^{*} \rightarrow U_{\alpha_{i}}^{*}$ such that, for each $u \in U_{\alpha_{i}}^{*}$,

$$
\dot{s}_{i} u \dot{s}_{i}=f_{i}(u) t_{i}(u) \dot{s}_{i} g_{i}(u)
$$

where $t_{i}(u) \in T$.

The proof can readily be adapted from [3, Lemma 2.1$]$.

We now begin the proof of Theorem 1.6, using induction on $l(w)$. It is clear, in all cases, that $U\left(w, w^{\prime}, w^{\prime \prime}\right)$ is independent of the choice of the cross section $\{\dot{x}\}$. In case $l(w)=1$, with $w=s_{i} \in S$, we have

$$
U\left(s_{i}, w^{\prime}, w^{\prime \prime}\right)= \begin{cases}1 & \text { if } s_{i} w^{\prime}=w^{\prime \prime}, l\left(s_{i} w^{\prime}\right)>l\left(w^{\prime}\right), \\ U_{\alpha_{i}} & \text { if } s_{i} w^{\prime}=w^{\prime \prime}, l\left(s_{i} w^{\prime}\right)<l\left(w^{\prime}\right), \\ U_{\alpha_{i}}^{*} & \text { if } w^{\prime}=w^{\prime \prime}, l\left(s_{i} w^{\prime}\right)<l\left(w^{\prime}\right),\end{cases}
$$


and is empty in all other cases. Using this information, Theorem 1.6 is easily verified in this case.

For the rest of the proof, $s_{l} \cdots s_{1}$ denotes a fixed reduced expression for $w$, with $l>1$. We assume the result holds for all triples $\left(s_{l-1} \cdots s_{1}, x, y\right)$, with $x, y \in W$, and note that $U\left(w, w^{\prime}, w^{\prime \prime}\right)$ is contained in the $k_{0}$-subgroup $U_{w}$, which can be factored

$$
U_{w}=U_{\alpha_{l}}{ }^{\dot{s}_{l}} U_{s_{l} w}
$$

with uniqueness, so that there exists an isomorphism of $k_{0}$-varieties $U_{w} \cong U_{\alpha_{l}} \times$ $U_{s_{l} w}$.

For a given triple $\left(s_{l} \cdots s_{1}, w^{\prime}, w^{\prime \prime}\right)$, we consider two cases.

Case $1 . l\left(s_{l} w^{\prime \prime}\right)<l\left(w^{\prime \prime}\right)$. In this case, by [4, proof of 2.14], the set of $K$-sequences $K=K\left(s_{l} \cdots s_{1}, w^{\prime}, w^{\prime \prime}\right)$ is partitioned, $K=K_{1} \cup K_{2}$, where $K_{1}$ consists of all sequences $\sigma=\left(\sigma_{l}, \ldots, \sigma_{1}, \sigma_{0}\right)$ with $\sigma^{\prime}=\left(\sigma_{l-1}, \ldots, \sigma_{1}, \sigma_{0}\right) \in K\left(s_{l-1} \cdots s_{1}, w^{\prime}, s_{l} w^{\prime \prime}\right)$ and $\sigma_{l} \sigma_{l-1}^{-1}=s_{l}$, while $K_{2}$ consists of all sequences $\tau=\left(\tau_{l}, \ldots, \tau_{1}, \tau_{0}\right)$ with $\tau^{\prime}=$ $\left(\tau_{l-1}, \ldots, \tau_{1}, \tau_{0}\right) \in K\left(s_{l-1} \cdots s_{1}, w^{\prime}, w^{\prime \prime}\right)$ and $\tau_{l} \tau_{l-1}^{-1}=1$.

For each sequence $\sigma \subset K_{1}$, we may assume the existence of $\left(U_{\sigma^{\prime}}, \Phi_{\sigma^{\prime}}, \Phi_{\sigma^{\prime}}^{*}\right)$ by the induction hypothesis, where $\sigma^{\prime} \in K\left(s_{l-1} \cdots s_{1}, w^{\prime}, s_{l} w^{\prime \prime}\right)$ is the part of $\sigma$ defined above. We then define

$$
U_{\sigma}=\dot{s}_{l}^{-1} U_{\sigma^{\prime}} \dot{s}_{l}, \quad \Phi_{\sigma}=s_{l} \Phi_{\sigma^{\prime}}, \quad \Phi_{\sigma}^{*}=s_{l} \Phi_{\sigma^{\prime}}^{*}
$$

so, in particular, $U_{\sigma}$ is a locally closed $k_{0}$-subvariety of $U_{w}$. We shall prove that the union $X_{1}$ of all subsets $\left\{U_{\sigma}: \sigma \in K_{1}\right\}$ is the part of $U\left(w, w^{\prime}, w^{\prime \prime}\right)$ contained in ${ }^{s_{l}} U_{s_{l} w}$ (see (2.2)). This follows since, as is easily checked, an element $u \in U_{s_{l} w}$ belongs to $U\left(s_{l} w, w^{\prime}, s_{l} w^{\prime \prime}\right)$ if and only if $\dot{s}_{l}^{-1} u \dot{s}_{l} \in U\left(w, w^{\prime}, w^{\prime \prime}\right)$. The part of the induction hypothesis giving the structure of each subset $U_{\sigma^{\prime}}$ in terms of the root subgroup $\left\{U_{\alpha}: \alpha \in \Phi_{\sigma^{\prime}}\right\}$ and $\left\{U_{\beta}: \beta \in \Phi_{\sigma^{\prime}}^{*}\right\}$ implies the corresponding result concerning the structure of $U_{\sigma}$ in terms of the root subgroups from roots in $\Phi_{\sigma}$ and $\Phi_{\sigma}^{*}$. This is clear since $U_{\sigma}$ is obtained from $U_{\sigma^{\prime}}$, and the root subgroups $\left\{U_{\alpha}: \alpha \in \Phi_{\sigma}\right\}$ and $\left\{U_{\beta}: \beta \in \Phi_{\sigma}^{*}\right\}$ are all obtained from those giving the structure of $U_{\sigma^{\prime}}$, by applying the inner automorphism by $\dot{s}_{l}$.

We now prove that the remaining part of $U\left(w, w^{\prime}, w^{\prime \prime}\right), X_{2}=U\left(w, w^{\prime}, w^{\prime \prime}\right)-X_{1}$, is the union of subsets associated with sequences $\tau \in K_{2}$. We first show that for an element $u_{w} \in U_{w}$ of the form $u_{w}=u \dot{s}_{l} u_{1} \dot{s}_{l}^{-1}$, with $u \in U_{\alpha_{l}}^{*}, u_{1} \in U_{s_{l} w}$ as in (2.2), we have

$$
u_{w} \in U\left(w, w^{\prime}, w^{\prime \prime}\right) \quad \text { if and only if } \quad \pi\left(g_{l}(u) u_{1}\right) \in U\left(s_{l} w, w^{\prime}, w^{\prime \prime}\right)
$$

where $\pi: U \rightarrow U_{s_{l} w}$ is the morphism of $k_{0}$-varieties defined by the factorization $U=U_{s_{l} w} U_{s_{l} w w_{0}}$ given by (1.2), and $g_{l}: U_{\alpha_{l}}^{*} \rightarrow U_{\alpha_{l}}^{*}$ is the automorphism of $k_{0^{-}}$ varieties defined by Lemma 2.1. To begin with, we have $u_{w} \in U\left(w, w^{\prime}, w^{\prime \prime}\right)$ if and only if

$$
\dot{s}_{l}^{-1} \dot{s}_{l} u \dot{s}_{l} u_{1} \dot{s}_{l}^{-1} \dot{w} B \cap \dot{w}^{\prime \prime} U_{\left(w^{\prime}\right)^{-1}}\left(\dot{w}^{\prime}\right)^{-1} \neq \varnothing .
$$

Using Lemma 2.1, this is equivalent to the statement

$$
\dot{s}_{l}^{-1} f_{l}(u) \dot{s}_{l} t_{l}(u) u_{1} \dot{s}_{l}^{-1} \dot{w} B \cap \dot{w}^{\prime \prime} U_{\left(w^{\prime}\right)^{-1}}\left(\dot{w}^{\prime}\right)^{-1} \neq \varnothing
$$

with $t=\dot{s}_{l}^{-1} t_{l}(u) \dot{s}_{l} \in T$ and $\dot{s}_{l}^{-1} f_{l}(u) \dot{s}_{l} \in U_{-\alpha_{l}}$.

In case $w^{\prime}\left(w^{\prime \prime}\right)^{-1} s_{i} \alpha_{i} \in \Phi^{-}$, we see that (2.4) holds if and only if

$$
g_{l}(u) u_{1}\left(s_{l} w\right)^{\cdot} B \cap \dot{w}^{\prime \prime} U_{\left(w^{\prime}\right)^{-1}}\left(\dot{w}^{\prime}\right)^{-1} \neq \varnothing
$$


since $w^{\prime \prime-1} U_{-\alpha_{l}} w^{\prime \prime} \leq U$ by the hypothesis of Case 1 , so the left factor of (2.4) can be absorbed in $U_{\left(w^{\prime}\right)^{-1}}$, while the element $t$ can be conjugated through the right-hand side, and then absorbed by $B$. Appying the factorization (1.2) for $s_{l} w$ to the element $g_{l}(u) u_{1}$, we obtain (2.3) in this case.

Now let $w^{\prime}\left(w^{\prime \prime}\right)^{-1} s_{l} \alpha_{l} \in \Phi^{+}$. Then (2.4) is equivalent to

$$
t g_{l}(u) u_{1} \dot{s}_{l}^{-1} \dot{w} B \cap \dot{w}^{\prime \prime} u^{*} U_{\left(w^{\prime}\right)^{-1}}\left(\dot{w}^{\prime}\right)^{-1} \neq \varnothing
$$

where

$$
u^{*}=\dot{w}^{\prime \prime-1} \dot{s}_{l}^{-1} f_{l}(u)^{-1} \dot{s}_{l} \dot{w}^{\prime \prime} \in U_{\left(w^{\prime}\right)^{-1} w_{0}} .
$$

Since $U_{\left(w^{\prime}\right)^{-1} w_{0}} U_{\left(w^{\prime}\right)^{-1}}=U_{\left(w^{\prime}\right)^{-1}} U_{\left(w^{\prime}\right)^{-1} w_{0}}$, and $\left(w^{\prime}\right)^{-1} U_{\left(w^{\prime}\right)^{-1} w_{0}} w^{\prime} \leq B$, we find that (2.6) is equivalent to (2.5), completing the proof of (2.3).

After some preliminary remarks, we shall apply (2.3) to define the subsets $\left\{U_{\tau}: \tau \in K_{2}\right\}$. Since $k_{0}$ is a perfect field and ${ }^{\dot{s}_{l}} U_{s_{l} w}$ is a closed $k_{0}$-subgroup of $U_{w}$, its complement $Z_{w}$ is an open subvariety of $U_{w}$ defined over $k_{0}$. The map $\left(u, u_{1}\right) \rightarrow u \dot{s}_{l} u_{1} \dot{s}_{l}^{-1}$ defines an isomorphism of $k_{0}$-varieties $U_{\alpha_{l}}^{*} \times U_{s_{l} w} \cong Z_{w}$. The $\operatorname{map} \phi: U_{\alpha_{l}}^{*} \times U_{s_{l} w} \rightarrow U_{s_{l} w}$ given by $\phi\left(u, u_{1}\right)=\pi\left(u u_{1}\right)$ for $u \in U_{\alpha_{l}}^{*}$ and $u_{1} \in U_{s_{l} w}$ is clearly a morphism of varieties over $k_{0}$. Now let $v \in U_{s_{l} w}$. For each fixed element $u \in U_{\alpha_{l}}^{*}$, it is easily verified using the factorization (1.2) that $u_{1}=\pi\left(u^{-1} v\right)$ is the unique element of $U_{s_{l} w}$ with the property that $\pi\left(u u_{1}\right)=v$. Combining these remarks, we see that the map $u s_{l} u_{1} s_{l}^{-1} \rightarrow \pi\left(g_{l}(u) u_{1}\right)$ is a surjective morphism of $k_{0}$-varieties from $Z_{w} \rightarrow U_{s_{l} w}$.

For each $K$-sequence $\tau \in K_{2}$, with $\tau=\left(\tau_{l}, \ldots, \tau_{0}\right)$, and $\tau^{\prime}=\left(\tau_{l-1}, \ldots, \tau_{0}\right)$ a $K$-sequence for the triple $\left(s_{l-1} \cdots s_{1}, w^{\prime}, w^{\prime \prime}\right)$, we define

$$
U_{\tau}=\left\{u \dot{s}_{l} u_{1} \dot{s}_{l}^{-1} \in U_{\alpha_{l}}^{*}{ }^{s_{l}} U_{s_{l} w}: \pi\left(g_{l}(u) u_{1}\right) \in U_{\tau^{\prime}}\right\}
$$

and

$$
\Phi_{\tau}=s_{l} \Phi_{\tau^{\prime}}, \quad \Phi_{\tau}^{*}=s_{l} \Phi_{\tau^{\prime}}^{*} \cup\left\{\alpha_{l}\right\} .
$$

By (2.3) and the induction hypothesis, it follows that $X_{2}=U\left(w, w^{\prime}, w^{\prime \prime}\right)-X_{1}$ is the disjoint union of the subsets $\left\{U_{\tau}\right\}, \tau \in K_{2}$.

Each subset $U_{\tau}$ is clearly a locally closed $k_{0}$-subvariety of $Z_{w}$, by the last statement in the paragraph preceding (2.7) and the induction hypothesis applied to $U_{\tau^{\prime}}$. Now let

$$
Y_{\tau}=\left\{\left(u, u_{1}\right) \in U_{\alpha_{l}}^{*} \times U_{s_{l} w}: \pi\left(u u_{1}\right) \in U_{\tau^{\prime}}\right\}
$$

Then $Y_{\tau}$ is a locally closed subvariety of $U_{\alpha}^{*} \times U_{s_{l} w}$ defined over $k_{0}$, and the map $u \dot{s}_{l} u_{1} \dot{s}_{l}^{-1} \rightarrow\left(g_{l}(u), u_{1}\right)$ defines an isomorphism of $k_{0}$-varieties $U_{\tau} \cong Y_{\tau}$. We next observe that the map $\xi: Y_{\tau} \rightarrow U_{\alpha_{l}}^{*} \times U_{\tau^{\prime}}$ defined by $\xi\left(u, u_{1}\right)=\left(u, \pi\left(u u_{1}\right)\right)$ is a surjective morphism of varieties over $k_{0}$. On the other hand, the preceding discussion shows that the map $\eta: U_{\alpha_{l}}^{*} \times U_{\tau^{\prime}} \rightarrow Y_{\tau}$ given by $\eta(u, v)=\left(u, \pi\left(u^{-1} v\right)\right)$ is a surjective morphism of $k_{0}$-varieties, and that $\xi$ and $\eta$ are inverses of each other. It follows that $Y_{\tau} \cong U_{\alpha_{l}}^{*} \times U_{\tau^{\prime}}$ as $k_{0}$-varieties, and hence the isomorphism $U_{\tau} \cong Y_{\tau}$ defines an isomorphism of $k_{0}$-varieties

$$
U_{\tau} \cong \prod_{\alpha \in \Phi_{\tau}} U_{\alpha} \times \prod_{\beta \in \phi_{\tau}^{*}} U_{\beta}^{*}
$$

using the definitions of $\Phi_{\tau}$ and $\Phi_{\tau}^{*}$ after (2.7). This completes the proof of the inductive step in Case 1. 
Case 2. $l\left(s_{l} w^{\prime \prime}\right)>l\left(w^{\prime \prime}\right)$. In this situation, each $K$-sequence $\tau=\left(\tau_{l}, \ldots, \tau_{0}\right)$ relative to $\left(s_{l} \cdots s_{1}, w^{\prime}, w^{\prime \prime}\right)$ has the property that $\tau_{l} \tau_{l-1}^{-1}=s_{l}$, and $\tau^{\prime}=\left(\tau_{l-1}, \ldots, \tau_{0}\right)$ is a $K$-sequence relative to $\left(s_{l-1} \cdots s_{1}, w^{\prime}, s_{l} w^{\prime \prime}\right)$. By [4, loc.cit.], this yields a bijection between the two sets of $K$-sequences. We shall prove that

$$
U\left(w, w^{\prime}, w^{\prime \prime}\right)=U_{\alpha_{l}} \dot{s}_{l} U\left(s_{l} w, w^{\prime}, s_{l} w^{\prime \prime}\right) \dot{s}_{l}^{-1} .
$$

Let $x \in U_{w}$, and write $x=u \dot{s}_{l} u_{1} \dot{s}_{l}^{-1}$, with $u \in U_{\alpha_{l}}, u_{1} \in U_{s_{l} w}$. Then $x \in$ $U\left(w, w^{\prime}, w^{\prime \prime}\right)$ if and only if

$$
u \dot{s}_{l} u_{1} \dot{s}_{l}^{-1} \dot{w} B \cap \dot{w}^{\prime \prime} U_{\left(w^{\prime}\right)^{-1}}\left(\dot{w}^{\prime}\right)^{-1} \neq \varnothing .
$$

Since $l\left(s_{l} w^{\prime \prime}\right)>l\left(w^{\prime \prime}\right)$, we have $\dot{w}^{\prime \prime-1} u \dot{w}^{\prime \prime} \in U$, and belongs either to $U_{\left(w^{\prime}\right)^{-1}}$ or to $U_{\left(w^{\prime}\right)^{-1} w_{0}}$. In either case, it is easily verified, using (1.2) if necessary, that $x \in U\left(w, w^{\prime}, w^{\prime \prime}\right)$ if and only if $u_{1} \in U\left(s_{l} w, w^{\prime}, s_{l} w^{\prime \prime}\right)$ which proves (2.8). Using (2.8) and the induction hypothesis, it is clear how to define the subsets $\left\{U_{\tau}\right\}$ for each $K$-sequence $\tau \in K\left(s_{l} \cdots s_{1}, w^{\prime}, w^{\prime \prime}\right)$, and corresponding sets of roots $\Phi_{\tau}$ and $\Phi_{\tau}^{*}$, so that the assertions of the theorem hold. This completes the proof of Theorem 1.6 .

The proof of Corollary 1.7 is obtained by following the steps of the proof of Theorem 1.6 for the group of rational points $G\left(k_{0}\right)$.

\section{REFERENCES}

1. A. Borel and J. Tits, Groupes réductifs, Inst. Hautes Études Sci. Publ. Math. 27 (1965), 55-150; ibid. 41 (1972), 253-276.

2. B. Chang, Decomposition of Gelfand-Graev characters of $\mathrm{GL}_{3}(q)$, Comm. Algebra 4 (1976), 375-401.

3. V. Deodhar, On some geometric aspects of Bruhat orderings. I. A finer decomposition of Bruhat cells, Invent. Math. 79 (1985), 499-511.

4. N. Kawanaka, Unipotent elements and characters of finite ('hevalley groups, Osaka J. Math. 12 (1975), 523-554.

5. R. Steinberg, Lectures on Chevalley groups, Yale University, 1967.

6. T. Yokonuma, Sur le commutant d'une représentation d'un groupe de ('hevalley fini, C. R. Acad. Sci. Paris 264 (1967), 433-436.

Department of Mathematics, University of Oregon, Eugene, Oregon 97403 